\title{
LOCATING GAS SOURCES USING A GAS SENSOR ARRAY
}

\section{Yukio Hiranaka and Hiro Yamasakit}

Dept. Electrical and Information Eng., Yamagata University, Yonezawa 992 Japan

†Dept. Mathematical Eng. and Info. Physics, The University of Tokyo, Bunkyo-ku, Tokyo 113 Japan

\begin{abstract}
Locating a gas source, such as a leak of fuel gas, is usually done using animal senses, but we are now developing techniques to do this job quickly, reliably and automatically. This is essentially requires the monitoring of a spatial field, using a two-dimensional array of gas sensors. The element sensors are of the semiconductor type. Because it is unusual for a gas field to be expressed by a simple diffusion model, gas source cannot be satisfactorily located using such a model. However, we have additionally used temporal data from each element sensor with promising results. The gas source can be inferred from the spatio-temporal information picked up by the sensors. We have carried out several modeling experiments with a 64-element sensor array and a test of its gas leak locating capability has yielded encouraging results. However, further work is required to improve the accuracy of the technique.
\end{abstract}

Key words gas sensor, two-dimensional array, gas source locating

A device to locate gas leaks is needed, because animal and human senses can not be reliably used in certain situations. An easy way to construct such a device is to use a gas sensor array. We have already build up a visualizing system for gaseous fields using such an array[1]. From this system, we can see the gas cloud and relative concentration in a real time manner. And we can locate the gas source on the screen. However, the sensor density of the array is limited, and the behavior of the gas cloud is influenced by many factors, such as air flow, direction of gas leak opening, vertical and horizontal distance between the source and a sensor, characteristics of the gas, obstacles in the field, etc. Thus it is not so easy to locate the source in real situations such as in chemical plants. Although experience and knowledge of the site are the usual means for overcoming these situations, we have tried to design a reliable method for localizing gas sources as described in this paper.

\section{APPARATUS}

The sensor array is comprised of 64 element sensors in a two dimensional square (8x8) shown in Fig.1[1]. Each element sensor is a $\mathrm{SnO}_{2}$ semiconductor type (Figaro, TGS701). Spacing between the sensors is $20 \mathrm{~cm}$ in both the vertical and the horizontal directions. The resistance of each sensor is measured in the form of voltage, and processed by a digital computer after AD conversion.

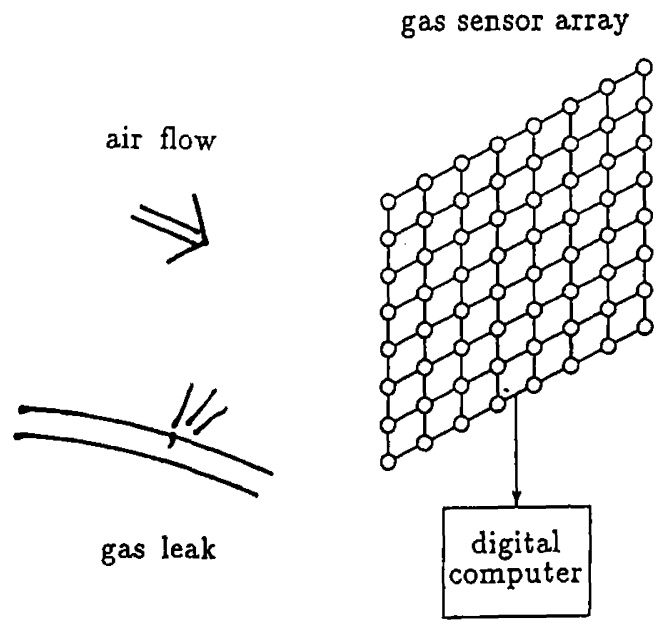

Fig.1. Gas source locating system using a gas sensor array. 
Gas concentration can be calculated by

$$
\log \frac{r}{r_{0}}=-m \log \frac{c+c_{1}}{c_{1}}
$$

where $r$ is the sensor resistance which is influenced by the gas concentration, $r_{0}$ is the resistance in gas free air, $m$ is a coefficient almost constant for any gas or sensors, $c$ is the gas concentration, $c_{1}$ is the characteristic value of the sensor which varies according to gas type and the operating surface temperature of the sensor[2]. Individual differences in each sensor are mainly accounted for by $r_{0}$. Therefore we used the value $r_{0} / r$ to represent the gas concentration.

\section{LOCATING METHOD}

Figure 2 shows an example of a visualized gas field. It is obvious that the center of the gas cloud in the figure probably does not indicate the gas source, because air flow in the field would shift the cloud. And air flow is not easily determined because of its fluctuating behavior.

First of all, let us consider a diffusion model. The general form of a diffusion equation in a three-dimensional space is

$$
\frac{\partial c}{\theta t}=D \Delta c
$$

where $t$ is time, $D$ is the diffusion coefficient specific to the gas component, and $\Delta$ is Laplacian (we assume that only one gas component exists in air, and that there is no absorption and no source when $t>0$ ).

The fundamental solution of equation (2) is

$$
c=\left(\frac{1}{4 \pi D t}\right)^{\frac{3}{2}} e^{-\frac{r^{2}}{4 D t}}
$$

where $r$ is the distance between the source point and the observation point. This shows the response to a single point impulse gas source $(\delta(r) \delta(t))$.

Figure 3 shows the relation between the concentration transient and the distance $r$ according to equation (3). It implies that we can determine the distance by the transient response of each sensor. However, actual data (Fig.4) do not fit this model; the time scale seems to be shorter than in the model response in Fig.3. This is an indication that the phenomena is not as simple as the above diffusion model.

Therefore, we need a more robust method to locate gas sources. As a second step, temporal data can be processed in conjunction with spatial information. A basic way to utilize spatial information is to measure the gas cloud at every sampling time and observe its movement. If the cloud moves in a certain direction while expanding in volume, we can identify the place of origin.

As there are only a very small number of sensors, we can calculate the area of the cloud from the interpolated gas field, as in Fig. 2. We made this interpolation using a bilinear equation

$$
f(\xi, \eta)=a+(b-a) \xi+(c-a) \eta+(a+d-b-c) \xi \eta
$$

where $\xi$ and $\eta$ are normalized $x, y$ coordinates in each rectangular element of the array and $a, b, c$ and $d$ are the gas concentrations at the vertices. Then, we could determine the area where the interpolated concentration is above a certain threshold and identify the centroid of the cloud.

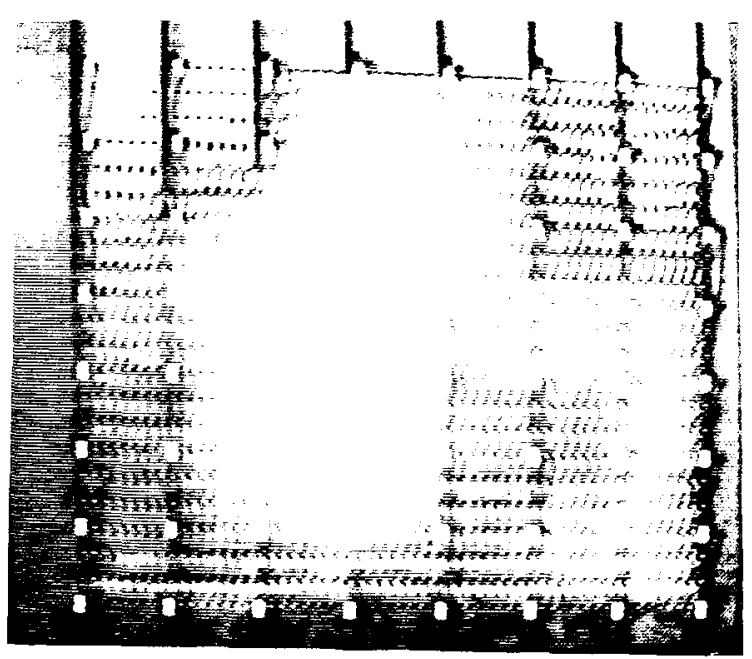

Fig.2. Typical example of a visualized gas field.

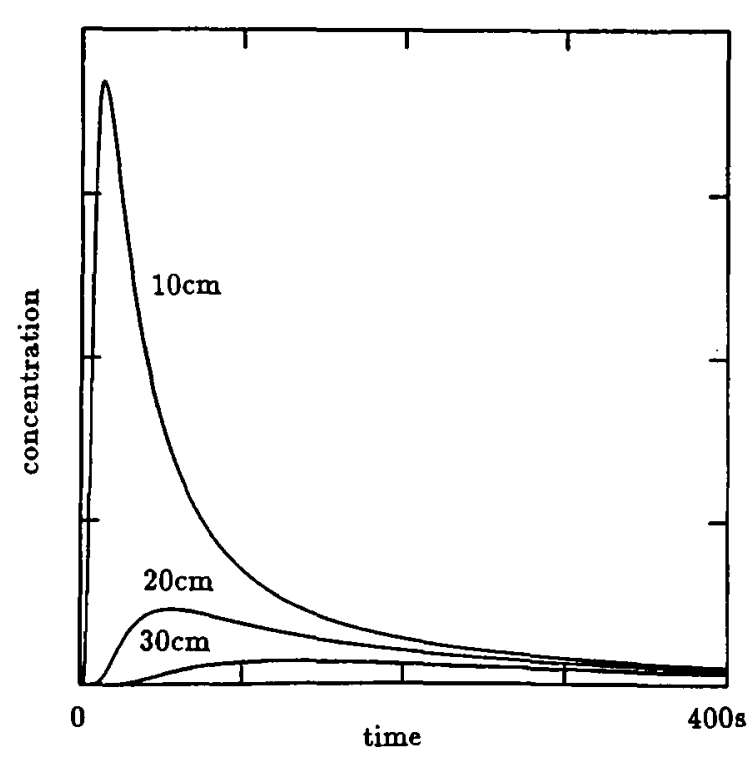

Fig.3. Gas concentration transients for ethyl alcohol predicted by the diffusion model for sensor-source distance of $10 \mathrm{~cm}, 20 \mathrm{~cm}$ and $30 \mathrm{~cm}$. 


\section{RESULTS}

The left parts of Figures 5 through 7 are typical examples of the mapping of clouds in a simple format. The path of the cloud's centroid is indicated by crosses. And cloud's size is represented by the area of the circles.

In Fig.5, a small bottle of liquid gas source (acetone) was placed at the center of the array which was vertical. The gas spread out high above the bottle after it was opened. It is easy to identify the gas source from Fig.5(a). In Fig.6, $60 \mathrm{ml}$ of ethyl alcohol gas was sprayed at the center of the array using a syringe $1.5 \mathrm{~m}$ from the vertical sensor array. The gas drifts to the upper right part of the array because of the air flow. However, if we inspect the figure closely, we can see that the cloud moved toward the center while enlarging its area and then moved back to its original location. Fig.7 shows similar experiment except that the distance from the array was $0.5 \mathrm{~m}$.

The right parts of Fig. 5-7 show the cloud area in relation to time. An almost linear expansion means that the source is at or near the array. Abrupt expansion means that the source is far away from the array. The inclination of the rising portion is roughly proportional to the distance of the source (the ratio is 1:4:10 for Fig.5, 6 and 7 , respectively). Although this relation should be studied further under various conditions, we can estimate the source distance under certain conditions.

\section{CONCLUSION}

We have performed several spatio-temporal exeriments concerning gas fields, and we have tested two methods for locating gas sources. A simple diffusion model is not good enough for determining the source, but using gas cloud analysis, it is possible to locate gas sources even outside the sensor array.

\section{REFERENCES}

[1] Y. Hiranaka, M. Some, M. Mishima and H. Yamasaki, "Visualizing system for spatial gas distribution," Proc. of SICE, pp.617-618 (1987).

[2] Y. Hiranaka and Masayuki Abe, "Compensation of no-gas response in gas identification using temperature change method with a semiconductor gas sensor," Proc. of SICE, pp.509-510 (1990).

[3] K.K. Carn, J. of Hazardous Materials, "Estimates of the mean concentration and variance for the Thorny Island phase I dense gas dispersion experiment," vol.16, pp.75-101 (1987).

[4] Y. Hiranaka and H. Yamasaki, "Measurement of Spatial Distribution Change of Gas Component by Using a Sensor Array," Technical Digest of the 8th Sensor Symposium, pp.177-180 (1989).

\begin{tabular}{|c|c|c|c|c|c|c|c|}
\hline$[0][0]$ & {$[0][1]$} & {$[0][2]$} & {$[0][3]$} & {$[0][4]$} & $18 \pi$ & {$[0)^{6]}$} & $\{0][7]$ \\
\hline$[1][0]$ & {$[1][1]$} & {$[1][2]$} & {$[1][3]$} & {$[1][4]$} & {$[1][5]$} & p]] & {$[1][7]$} \\
\hline$[2][0]$ & {$[2][1]$} & {$[2][2]$} & {$[2][3]$} & {$[2][4]$} & {$[2][5]$} & {$[\phi][Q]$} & {$[2][7]$} \\
\hline$[3][0]$ & {$[3][1]$} & {$[3][2]$} & {$[3][3]$} & {$[3][4]$} & {$[3][5]$} & {$[3][6]$} & $\{3][7]$ \\
\hline$[4][0]$ & {$[4][1]$} & {$[4][2]$} & {$[4][3]$} & {$[4][4]$} & {$[4][5]$} & {$[4][8]$} & {$[4][7]$} \\
\hline$[5][0]$ & {$[5][1]$} & {$[5][2]$} & {$[5][3]$} & {$[5][4]$} & {$[5][5]$} & {$[5][8]$} & {$[5][7]$} \\
\hline$[6][0]$ & {$[8][1]$} & {$[8][2]$} & {$[6][3]$} & {$[6][4]$} & {$[8][5]$} & {$[6][6]$} & {$[8][7]$} \\
\hline$[7][0]$ & {$[7][1]$} & {$[7][2]$} & {$[7][3]$} & {$[7][4]$} & {$[7][5]$} & {$[7][6]$} & {$[7][7]$} \\
\hline
\end{tabular}

Fig.4. An example of array data. The ordinate of each cell in the array shows $r_{0} / r$ and the abscissa represents a 15 second time span. 


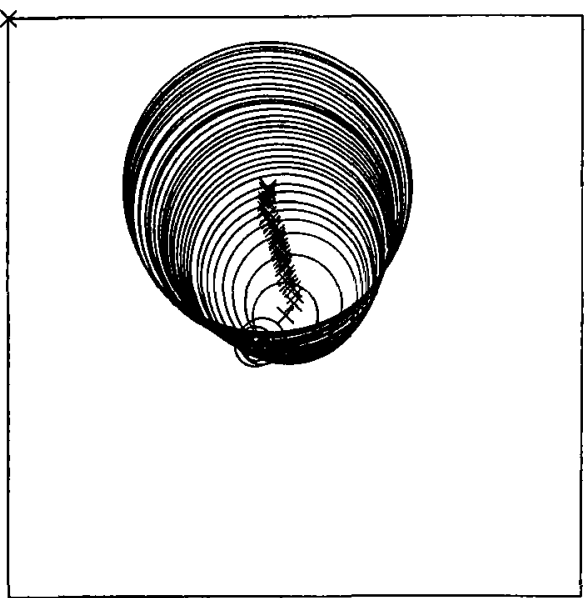

(a)

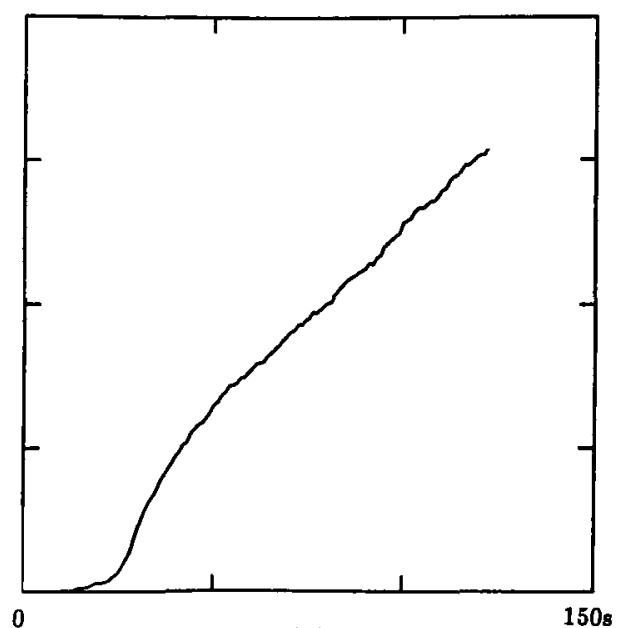

(b)

Fig.5. The result of gas exposure applied as a step time function with the gas source at the center of the array on the array plane. (a) shows the movement of the centroid (indicated by crosses) and the area (circle) of the gas cloud with time. (b) shows the change in gas cloud area with time.

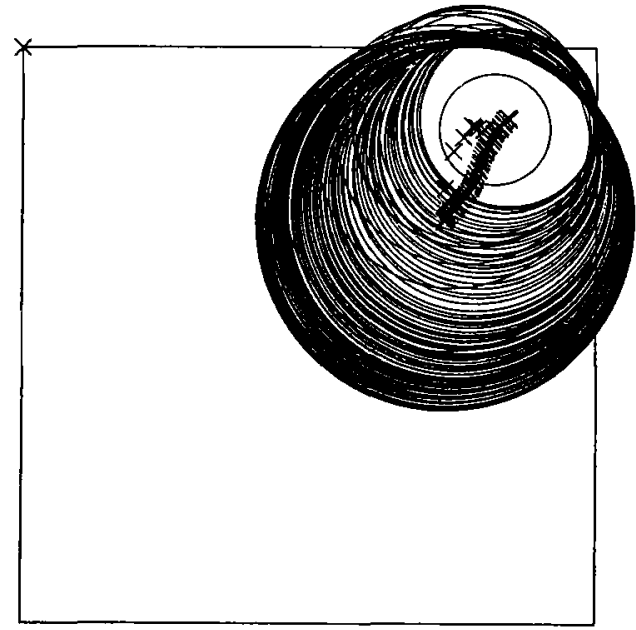

(a)

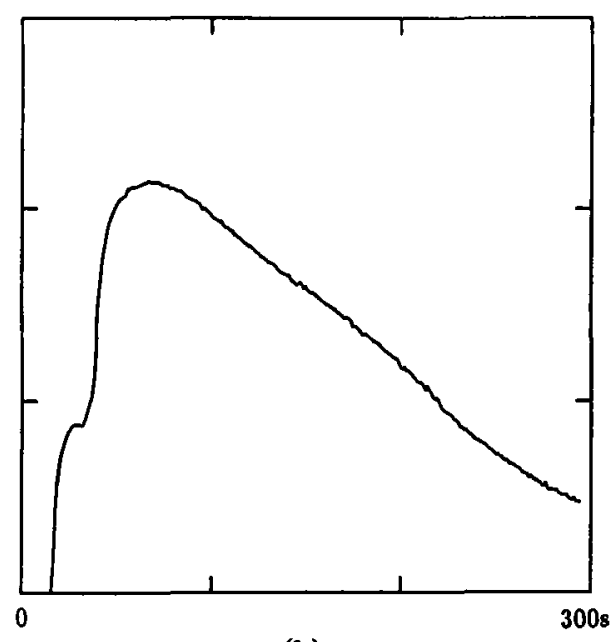

(b)

Fig.6. The result of gas exposure applied as an impulse function with the gas source located 1.5m in front of the center of the array.

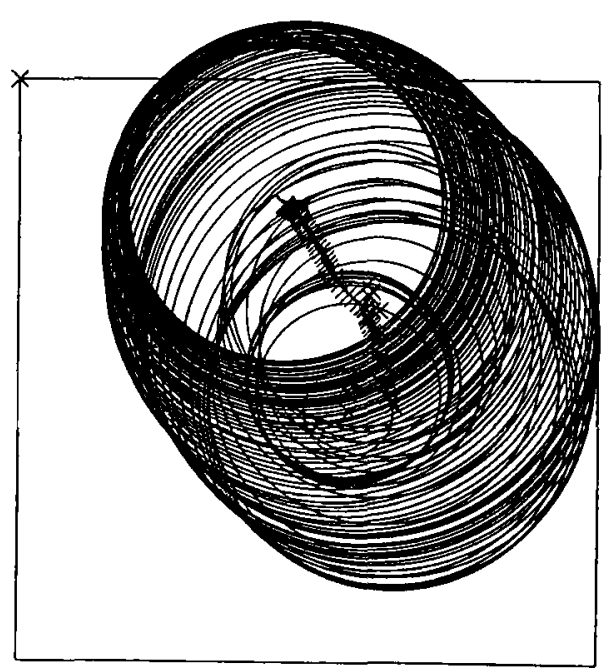

(a)

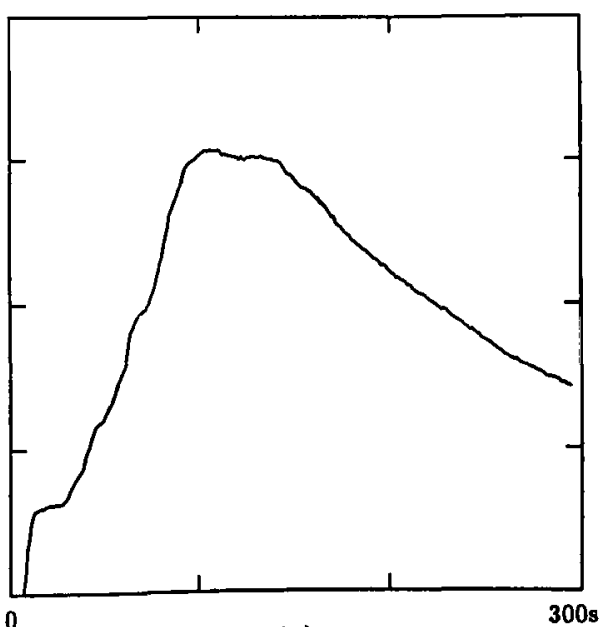

(b)

Fig.7. The result of gas exposure applied as an impulse function with the gas source located $0.5 \mathrm{~m}$ in front of the center of the array. 